\title{
Initial value problems in linear integral operator equations*
}

\author{
L.P. Castro, M.M. Rodrigues and S. Saitoh
}

\begin{abstract}
For some general linear integral operator equations, we investigate consequent initial value problems by using the theory of reproducing kernels. A new method is proposed which - in particular - generates a new field among initial value problems, linear integral operators, eigenfunctions and values, integral transforms and reproducing kernels. In particular, examples are worked out for the integral equations of Lalesco-Picard, Dixon and Tricomi types.
\end{abstract}

Keywords: Integral transform, reproducing kernel, isometric mapping, inversion formula, initial value problem, eigenfunction, eigenvalue, Fourier integral transform, inverse problem, Lalesco-Picard equation, Dixon equation, Tricomi equation.

MSC2010: Primary 45C05; Secondary 32A30, 42A38, 45A05, 45D05, 45E05, 45P05, 46E22, 47A05.

\section{Introduction}

Despite the fact that initial value problems for differential equations, and consequent integral equations, have already a long and rich history, there is still the need in different cases to find out additional and more suitable spaces where their solutions can be interpreted and used in an appropriated way. Sometimes, finding new

\footnotetext{
* Accepted author's manuscript (AAM) published in [L.P. Castro, M.M. Rodrigues, S. Saitoh: Initial value problems in linear integral operator equations, Th.M. Rassias, L. Tóth (edts), Springer Optimization and Its Applications, Vol. 94, 175-188, Springer International Publishing, New York, 2014] [DOI: 10.1007/978-3-319-06554-0_7]. The final publication is available at Springer via http://link.springer.com/chapter/10.1007/978-3-319-06554-0_7

L.P. Castro, M.M. Rodrigues and S. Saitoh

CIDMA-Center for Research and Development in Mathematics and Applications, Department of Mathematics, University of Aveiro, 3810-193 Aveiro, Portugal, e-mail: castro@ua.pt; mrodrigues@ua.pt; saburou.saitoh@gmail.com
} 
frameworks for those solutions leads even to the discover of completely new solutions which could not be reached in a somehow more "classical" and known setting. This is just one of the reasons why it is still well appropriate to continue to perform research in such a classical field. Moreover, additional knowledge about the solutions is also very welcome - even in cases where we know already a great variety of solutions. This is the case of the study of different kinds of stability which is, e.g., highly relevant when we would like to apply numerical methods. Within this spirit, we would like to consider initial value problems in linear integral operator equations by using reproducing kernel Hilbert space machinery [1, 12, 13, 14].

Having those general goals in mind, in [4] the authors proposed a general method for the existence and construction of the solution of the following initial problem

$$
\left(\partial_{t}+L_{x}\right) u_{f}(t, x)=0, \quad t>0
$$

satisfying the initial value condition

$$
u_{f}(0, x)=f(x)
$$

for some general linear operator $L_{x}$ on a certain function space, and on some domain, by using the theory of reproducing kernels.

Here, we consider a general linear integral equation

$$
I_{x} u(x)=0
$$

and we assume that the eigenfunctions $L_{v}$ and values $v$ are known; that is,

$$
I_{x} L_{v}(x)=v L_{v}(x)
$$

Then, note that the functions

$$
\exp (-v t) L_{v}(x)
$$

are the solutions of the operator equation

$$
\left(\partial_{t}+I_{x}\right) u(t, x)=0
$$

In order to consider a fully general sum, in the case that $v$ are positive reals, we shall consider the kernel form, for a nonnegative continuous function $\rho$,

$$
\mathscr{K}_{t}(x, y ; \rho)=\int_{0}^{+\infty} \exp \{-v t\} L_{v}(x) \overline{L_{v}(y)} \rho(v) d v .
$$

Of course, in here, we are considering the integral with absolutely convergence for the kernel form.

The fully general solutions of the equation (6) may be represented in the integral form

$$
u(t, x)=\int_{0}^{+\infty} \exp \{-v t\} L_{v}(x) F(v) \rho(v) d v,
$$

for the functions $F$ satisfying 


$$
\int_{0}^{+\infty} \exp \{-v t\}|F(v)|^{2} \rho(v) d v<\infty .
$$

Then, the solution $u(t, x)$ of (6) satisfying the initial condition

$$
u(0, x)=F(x)
$$

will be obtained by $t \rightarrow+0$ in (8) with a natural meaning. However, this point will be very delicate and we will need to consider some deep and beautiful structure. Here, (7) is a reproducing kernel and in order to analyze the logic above, we will need the theory of reproducing kernels within an essential (and beautiful) way. Indeed, in order to construct natural solutions of (1)-(2), we will need a new framework and function space.

\section{Preliminaries on linear mappings and inversions}

In order to analyze the integral transform (8) and in view to set the basic background for our purpose, we will need the essence of the theory of reproducing kernels.

We are interested in the integral transforms (8) in the framework of Hilbert spaces. Of course, we are interested in the characterization of the image functions, the consequent isometric identity like the Parseval identity and the inversion formula, basically. For these general and fundamental problems, we have a unified and fundamental method and concept in the general situation. Namely, following $[12,13,14]$, we shall recall a general theory for linear mappings in the framework of Hilbert spaces.

Let $\mathscr{H}$ be a Hilbert (possibly finite-dimensional) space. Let $E$ be an abstract set and $\mathbf{h}$ be a Hilbert $\mathscr{H}$-valued function on $E$. Then, we shall consider the linear transform

$$
f(x)=(\mathbf{f}, \mathbf{h}(x))_{\mathscr{H}}, \quad \mathbf{f} \in \mathscr{H},
$$

from $\mathscr{H}$ into the linear space $\mathscr{F}(E)$ comprising all the complex valued functions on $E$. In order to investigate the linear mapping (11), we form a positive definite quadratic form function $K(x, y)$ on $E \times E$ defined by

$$
K(x, y)=(\mathbf{h}(y), \mathbf{h}(x))_{\mathscr{H}} \quad \text { on } \quad E \times E .
$$

A complex-valued function $k: E \times E \rightarrow \mathbb{C}$ is called a positive definite quadratic form function on the set $E$, or shortly, positive definite function, when it satisfies the property that, for an arbitrary function $X: E \rightarrow \mathbb{C}$ and any finite subset $F$ of $E$,

$$
\sum_{x, y \in F} \overline{X(x)} X(y) k(x, y) \geq 0 .
$$


By the fundamental theorem, we know that for any positive definite quadratic form function $K$, there exists a uniquely determined reproducing kernel Hilbert space admitting the reproducing property.

Then, we obtain the following fundamental result.

Proposition 1 (cf. $[12,13,14])$.

(A) The range of the linear mapping (11) by $\mathscr{H}$ is characterized as the reproducing kernel Hilbert space $H_{K}(E)$ admitting the reproducing kernel $K(x, y)$ whose characterization is given by the two properties: $(i) K(\cdot, y) \in H_{K}(E)$ for any $y \in E$ and,

(ii) for any $f \in H_{K}(E)$ and for any $x \in E,(f(\cdot), K(\cdot x))_{H_{K}(E)}=f(x)$.

(B) It holds

$$
\|f\|_{H_{K}(E)} \leq\|\mathbf{f}\|_{\mathscr{H}}
$$

Here, for any member $f$ of $H_{K}(E)$ there exists a uniquely determined $\mathbf{f}^{*} \in \mathscr{H}$ satisfying

$$
f(x)=\left(\mathbf{f}^{*}, \mathbf{h}(x)\right)_{\mathscr{H}} \quad \text { on } E
$$

and

$$
\|f\|_{H_{K}(E)}=\left\|\mathbf{f}^{*}\right\|_{\mathscr{H}} .
$$

(C) We have the inversion formula in (11) in the form

$$
f \mapsto \mathbf{f}^{*}
$$

in $(B)$ by using the reproducing kernel Hilbert space $H_{K}(E)$.

However, in general, this formula (13) is not obvious. Consequently, case by case, we need different arguments to analyse it. See [13] and [14] for the details and applications. Recently, however, we obtained a very general inversion formula, based on the so-called Aveiro Discretization Method in Mathematics (cf. [2]), by using the ultimate realization of reproducing kernel Hilbert spaces that is introduced simply in the last section. In this paper, however, in order to give prototype examples with analytical nature, we shall consider the following global inversion formula in the general situation with natural assumptions.

Here we consider a concrete case of Proposition 1. In order to derive a general inversion formula widely applicable in analysis, we assume that $\mathscr{H}=L^{2}(I, d m)$ and that $H_{K}(E)$ is a closed subspace of $L^{2}(E, d \mu)$. Furthermore, below we assume that $(I, \mathscr{I}, d m)$ and $(E, \mathscr{E}, d \mu)$ are both $\sigma$-finite measure spaces and that

$$
H_{K}(E) \hookrightarrow L^{2}(E, d \mu) .
$$

Suppose that we are given a measurable function $h: I \times E \rightarrow \mathbb{C}$ satisfying $h_{y}=$ $h(\cdot, y) \in L^{2}(I, d m)$ for all $y \in E$. Let us set

$$
K(x, y) \equiv\left\langle h_{y}, h_{x}\right\rangle_{L^{2}(I, d m)} .
$$

As we have established in Proposition 1, we have

$$
H_{K}(E) \equiv\left\{f \in \mathscr{F}(E): f(x)=\left\langle F, h_{x}\right\rangle_{L^{2}(I, d m)} \text { for some } F \in \mathscr{H}\right\} .
$$


Let us now define

$$
L: \mathscr{H} \rightarrow H_{K}(E)\left(\hookrightarrow L^{2}(E, d \mu)\right)
$$

by

$$
L F(x) \equiv\left\langle F, h_{x}\right\rangle_{L^{2}(I, d m)}=\int_{I} F(\lambda) \overline{h(\lambda, x)} d m(\lambda), \quad x \in E,
$$

for $F \in \mathscr{H}=L^{2}(I, d m)$, keeping in mind (14). Observe that $L F \in H_{K}(E)$.

The next result will serve to the inversion formula.

Proposition 2 (cf. [13]). Assume that $\left\{E_{N}\right\}_{N=1}^{\infty}$ is an increasing sequence of measurable subsets in E such that

$$
\bigcup_{N=1}^{\infty} E_{N}=E
$$

and that

$$
\int_{I \times E_{N}}|h(\lambda, x)|^{2} d m(\lambda) d \mu(x)<\infty
$$

for all $N \in \mathbb{N}$. Then we have

$$
L^{*} f(\lambda)\left(=\lim _{N \rightarrow \infty}\left(L^{*}\left[\chi_{E_{N}} f\right]\right)(\lambda)\right)=\lim _{N \rightarrow \infty} \int_{E_{N}} f(x) h(\lambda, x) d \mu(x)
$$

for all $f \in L^{2}(I, d \mu)$ in the topology of $\mathscr{H}=L^{2}(I, d m)$. Here, $L^{*} f$ is the adjoint operator of $L$, but it represents the inversion with the minimum norm for $f \in H_{K}(E)$.

Moreover, in this Proposition 2, we see that -in a very natural way- the inversion formula may be given in the strong convergence in the space $\mathscr{H}=L^{2}(I, d m)$.

\section{Main result}

Following the general theory in Section 2, we shall now build our results. Without loss of generality, we will assume that $v$ is on the positive real line.

Then, we form the reproducing kernel

$$
\mathscr{K}(x, y ; \rho)=\int_{0}^{+\infty} L_{v}(x) \overline{L_{v}(y)} \rho(v) d v, \quad t>0,
$$

and consider the reproducing kernel Hilbert space $H_{\mathscr{K}(\rho)}\left(\mathbb{R}^{+}\right)$admitting the kernel $\mathscr{K}(x, y ; \rho)$. Here, we assume that the kernel form converges in the absolute sense. In particular, note that

$$
\mathscr{K}_{t}(x, y ; \rho) \in H_{\mathscr{K}(\rho)}\left(\mathbb{R}^{+}\right), \quad y>0 .
$$


Then, we obtain the main theorem in this paper:

Theorem 1. For any member $f \in H_{\mathscr{K}(\rho)}\left(\mathbb{R}^{+}\right)$, the solution $u_{f}(t, x)$ of the initial value problem, for $t>0$,

$$
\left(\partial_{t}+I_{x}\right) u_{f}(t, x)=0,
$$

satisfying the initial value condition

$$
u_{f}(0, x)=f(x)
$$

exists and it is given by

$$
u_{f}(t, x)=\left(f(\cdot), \mathscr{K}_{t}(\cdot, x ; \rho)\right)_{H_{\mathscr{K}(\rho)}}\left(\mathbb{R}^{+}\right) \cdot
$$

Here, the meaning of the initial value (24) is given by

$$
\begin{aligned}
\lim _{t \rightarrow+0} u_{f}(t, x) & =\lim _{t \rightarrow+0}\left(f(\cdot), \mathscr{K}_{t}(\cdot, x ; \rho)\right)_{H_{\mathscr{K}}(\rho)}\left(\mathbb{R}^{+}\right) \\
& =(f(\cdot), \mathscr{K}(\cdot, x ; \rho))_{H_{\mathscr{K}}(\rho)}\left(\mathbb{R}^{+}\right) \\
& =f(x),
\end{aligned}
$$

whose existence is ensured and the limit is the uniformly convergence on any subset of $\mathbb{R}^{+}$such that $\mathscr{K}(x, x ; \rho)$ is bounded.

The uniqueness property of the initial value problem is depending on the completeness of the family of functions

$$
\left\{\mathscr{K}_{t}(\cdot, x ; \rho): x \in \mathbb{R}^{+}\right\}
$$

in $H_{\mathscr{K}(\rho)}\left(\mathbb{R}^{+}\right)$.

Before starting with the proof of the Theorem some remarks are in order. In our theorem, the complete property of the solutions $u_{f}(t, x)$ of (23) and (24) satisfying the initial value $f$ may be derived by the reproducing kernel Hilbert space admitting the kernel

$$
k(x, t ; y, \tau ; \rho):=\left(\mathscr{K}_{\tau}(\cdot, y ; \rho), \mathscr{K}_{t}(\cdot, x ; \rho)\right)_{H_{\mathscr{K}(\rho)}\left(\mathbb{R}^{+}\right)} .
$$

In our method, we see that the existence problem of the initial value problem is based on the eigenfunctions and we are constructing the desired solution satisfying the desired initial condition. For a larger knowledge for the eigenfunctions we can consider a more general initial value problem.

Furthermore, by considering the linear mapping of (25) with various situations, we will be able to obtain various inverse problems looking for the initial values $f$ from the various output data of $u_{f}(t, x)$.

Proof (of Theorem 1). In first place, note that the kernel $\mathscr{K}_{t}(x, y ; \rho)$ satisfies the operator equation (23) for any fixed $y$, because the functions

$$
\exp \{-v t\} L_{v}(x)
$$


satisfy the operator equation and it is the summation. Similarly, the function $u_{f}(t, x)$ defined by (25) is the solution of the operator equation (23).

Secondly, in order to see the initial value problem, we note the important general property

$$
\mathscr{K}_{t}(x, y ; \rho) \ll \mathscr{K}(x, y ; \rho) ;
$$

that is, $\mathscr{K}(x, y ; \rho)-\mathscr{K}_{t}(x, y ; \rho)$ is a positive definite quadratic form function. Moreover, we have

$$
H_{\mathscr{K}_{t}(\rho)} \subset H_{\mathscr{K}(\rho)}\left(\mathbb{R}^{+}\right)
$$

and for any function $f \in H_{\mathscr{K}_{t}}(\rho)$

$$
\|f\|_{H_{\mathscr{K}(\rho)}\left(\mathbb{R}^{+}\right)}=\lim _{t \rightarrow+0}\|f\|_{H_{\mathscr{K}_{t}(\rho)}}
$$

in the sense of non-decreasing norm convergence (see [1]). In order to see the crucial point in (26), note that

$$
\begin{aligned}
\| \mathscr{K}(y, x ; \rho) & -\mathscr{K}_{t}(y, x ; \rho) \|_{H_{\mathscr{K}}(\rho)}^{2}\left(\mathbb{R}^{+}\right) \\
& =\mathscr{K}(x, x ; \rho)-2 \mathscr{K}_{t}(x, x ; \rho)+\left\|\mathscr{K}_{t}(y, x ; \rho)\right\|_{H_{\mathscr{K}}(\rho)}^{2}\left(\mathbb{R}^{+}\right) \\
& \leq \mathscr{K}(x, x ; \rho)-2 \mathscr{K}_{t}(x, x ; \rho)+\left\|\mathscr{K}_{t}(y, x ; \rho)\right\|_{H_{\mathscr{K}_{t}(\rho)}}^{2} \\
& =\mathscr{K}(x, x ; \rho)-\mathscr{K}_{t}(x, x ; \rho),
\end{aligned}
$$

that converses to zero as $t \rightarrow+0$. We thus obtain the desired limit property in the theorem.

Finally, the uniqueness property of the initial value problem follows from (25) easily.

\section{Examples}

At first, we shall consider the typical and famous Lalesco-Picard equation:

$$
y(x)-\lambda \int_{-\infty}^{\infty} e^{-|x-t|} y(t) d t=0, \quad \lambda>0 .
$$

Then, we know the general solution

$$
y(x)=C_{1} \exp (x \sqrt{1-2 \lambda})+C_{2} \exp (-x \sqrt{1-2 \lambda}), \quad 0<\lambda<\frac{1}{2},
$$

and

$$
y(x)=C_{1} \cos (x \sqrt{1-2 \lambda})+C_{2} \sin (x \sqrt{1-2 \lambda}), \quad \frac{1}{2}<\lambda,
$$

$($ see $[7,11])$. 
From the results, we can consider the four eigenfunctions and eigenvalues groups; so, without loss of generality, we shall consider the case, for

$$
y_{\lambda}(x)=\exp (-x \sqrt{1-2 \lambda})
$$

in which we have

$$
\int_{-\infty}^{\infty} e^{-|x-t|} y_{\lambda}(t) d t=\frac{1}{\lambda} y_{\lambda}(x), \quad 0<\lambda<\frac{1}{2} .
$$

Therefore, by a suitable weight $\rho$, we shall consider the reproducing kernel

$$
\int_{0}^{1 / 2} \exp (-y \sqrt{1-2 \lambda}) \exp (-x \sqrt{1-2 \lambda}) \rho(\lambda) d \lambda
$$

Note that we can consider many weights $\rho$, however, as the simplest case, we obtain the reproducing kernel

$$
\begin{aligned}
K(x, y) & =\int_{0}^{1 / 2} \exp (-y \sqrt{1-2 \lambda}) \exp (-x \sqrt{1-2 \lambda}) \frac{1}{\sqrt{1-2 \lambda}} d \lambda \\
& =\frac{1}{x+y}\left(1-e^{-x} e^{-y}\right) .
\end{aligned}
$$

Now, we are interested in the integral transform

$$
f(x)=\int_{0}^{1 / 2} F(\lambda) \exp (-x \sqrt{1-2 \lambda}) \frac{1}{\sqrt{1-2 \lambda}} d \lambda
$$

for the functions $F$ satisfying the conditions

$$
\int_{0}^{1 / 2}|F(\lambda)|^{2} \frac{1}{\sqrt{1-2 \lambda}} d \lambda<\infty
$$

Note that for the kernel form

$$
\frac{1}{z+\bar{u}}, \quad z=x+i y
$$

on the right half complex plane, this reproducing kernel is the Szegö kernel and for the image of the integral transform

$$
f(z)=\int_{0}^{\infty} e^{-\lambda z} F(\lambda) d \lambda,
$$

for the $L_{2}(0, \infty)$ functions $F(\lambda)$, we obtain the isometric identity

$$
\frac{1}{2 \pi} \int_{-\infty}^{+\infty}|f(i y)|^{2} d y=\int_{0}^{\infty}|F(\lambda)|^{2} d \lambda
$$


Here, $f(i y)$ means the Fatou's non-tangential boundary values of the Szegö space of analytic functions on the right hand half complex plane.

From the relation for analytic extension

$$
K(z, \bar{u}) \ll \frac{1}{z+\bar{u}}
$$

(in the sense that the right hand side minus the left hand side is a positive definite quadratic form function), we see that the admissible reproducing kernel Hilbert spaces $H_{K}$ and $H_{S}$ have the inclusion relation as functions

$$
H_{K} \subset H_{S}
$$

and we have the norm inequality, for any $f \in H_{K}$,

$$
\|f\|_{H_{S}} \leq\|f\|_{H_{K}}
$$

The space $H_{K}$ is a subspace of the Szegö space $H_{S}$ and so we can use the Szegö space $H_{S}$ for the isometric identity and inversion formula. For extra details on these general properties, see [13]. As the conclusions, we see that the image $f(x)$ of the integral transform (37) is extensible analytically onto the right half complex plane as $f(z), z=x+i y$, and we obtain the norm inequalities

$$
\int_{0}^{1 / 2}|F(\lambda)|^{2} \frac{1}{\sqrt{1-2 \lambda}} d \lambda \leq \frac{1}{2 \pi} \int_{-\infty}^{+\infty}|f(i y)|^{2} d y .
$$

Furthermore, we obtain the inversion formula in the space satisfying (38)

$$
F(\lambda)=\int_{-\infty}^{\infty} f(i y) \exp (-i y \sqrt{1-2 \lambda}) d y .
$$

For other eigenfunctions, we can obtain similar results. For other weighted functions, we can obtain more complicated results; see [13] for consequent details.

Next, as a typical example of Volterra integral equations, we shall consider, the Dixon's equation

$$
y(x)-\lambda \int_{0}^{x} \frac{y(t) d t}{x+t}=f(x), \quad \lambda>0 .
$$

For the homogeneous case of $f \equiv 0$, we know the solutions

$$
y(x)=C x^{\beta} ; \quad \beta>-1,
$$

where

$$
\lambda=\frac{1}{I(\beta)} ; \quad I(\beta)=\int_{0}^{1} \frac{\xi^{\beta} d \xi}{1+\xi},
$$

(cf. [11], p. 136). Therefore, for the integral operator

$$
\int_{0}^{x} \frac{y(t) d t}{x+t}
$$


we have the eigenfunctions and eigenvalues

$$
y(t)=I(\lambda) t^{\lambda}, \quad \lambda>-1
$$

Thus, we obtain the related reproducing kernel, for $0<x, y<1$, for example,

$$
\begin{aligned}
K(x, y) & =\int_{-1}^{\infty} x^{\lambda} y^{\lambda} d \lambda \\
& =-\frac{1}{\ln x y} \frac{1}{x y}
\end{aligned}
$$

The property of the integral transform

$$
f(x)=\int_{-1}^{\infty} x^{\lambda} F(\lambda) d \lambda
$$

for the functions $F$ satisfying

$$
\int_{-1}^{\infty}|F(\lambda)|^{2} d \lambda<\infty
$$

will be involved. However, we see that the image $f$ is extensible analytically onto the complex plane cutted by the half real line $(-\infty, 0]$.

By the complex conformal mapping $W=\log z$, the image $f(z)=f\left(e^{w}\right)$ of (53) may be discussed by the Szegö space on the strip domain

$$
\left\{\mathfrak{I} w<\frac{\pi}{2}\right\}
$$

At last, as a typical example of singular integral equations, we shall consider the Tricomi equation

$$
y(x)-\lambda \int_{0}^{1}\left(\frac{1}{t-x}+\frac{1}{x+t-2 x t}\right) y(t) d t=f(x), \quad \lambda>0 .
$$

For the homogeneous case of $f \equiv 0$, we know the solutions

$$
y(x)=C \frac{(1-x)^{\beta}}{x^{1+\beta}} ; \quad \tan \frac{\beta \pi}{2}=\lambda \pi, \quad-2<\beta<0
$$

(cf. [11], p. 769). Therefore, for the integral operator

$$
\int_{0}^{1}\left(\frac{1}{t-x}+\frac{1}{x+t-2 x t}\right) y(t) d t
$$

we have the eigenfunctions and eigenvalues

$$
y(t)=\frac{1}{\lambda} \frac{(1-x)^{\frac{2}{\pi} \arctan \pi \lambda}}{x^{1+\frac{2}{\pi} \arctan \pi \lambda}} .
$$


Therefore, we obtain the related reproducing kernel, for $0<x, y<1$ and for example, for $0<\lambda<\infty$ :

$$
\begin{aligned}
K(x, y) & =\int_{0}^{\infty} \frac{(1-x)^{\frac{2}{\pi} \arctan \pi \lambda}}{x^{1+\frac{2}{\pi} \arctan \pi \lambda}} \frac{(1-y)^{\frac{2}{\pi} \arctan \pi} \pi}{y^{1+\frac{2}{\pi} \arctan \pi \lambda}} d \lambda \\
& =\frac{1}{2} \int_{0}^{1} \frac{(1-x)^{\xi}}{x^{1+\xi}} \frac{(1-y)^{\xi}}{y^{1+\xi}} \sec ^{2} \frac{\pi \xi}{2} d \xi .
\end{aligned}
$$

As typical integral equations, we stated the above three integral equations. However, we can consider many and many different integral equations, and the eigenfunctions structures will be mysterious deep and we are requested to analyze their structures and the corresponding integral transforms. Furthermore, we are particularly interested in kernel form integrals. To this end, the great book [11] presents a huge range of possibilities.

\section{Concrete realization of the reproducing kernel Hilbert paces}

In Section 4, we can consider many concrete forms of the reproducing kernels and among those we have very complicated structures of the related reproducing kernel Hilbert spaces. Even just from the point of view of the theory of reproducing kernels, their realizations will give interesting research topics that are requested separate papers.

We were able to realize the important reproducing kernel Hilbert spaces concretely and analytically. However, for many kernels their realizations will be complicated. Despite this difficulty, it is clear that the concrete forms of the reproducing kernels will be very important and interested by themselves.

Meanwhile, we are also interested in the kernel forms $\mathscr{K}_{t}$ and $k$. These calculations will create a new and large field in integral formulas.

As explained, we have to analyze and realize the corresponding reproducing kernel Hilbert spaces. However, we can also apply quite general formula by the Aveiro discretization method exposed in $[2,3]$. In these papers, numerical experiments are also given based on the following result:

Proposition 3. (Ultimate realization of reproducing kernel Hilbert spaces). In our general situation and for a uniqueness set $\left\{p_{j}\right\}$ for the reproducing kernel Hilbert space $H_{K}$ of the set $E$ satisfying the linearly independence of $K\left(\cdot, p_{j}\right)$ for any finite number of the points $p_{j}$, we obtain

$$
\|f\|_{H_{K}}^{2}=\left\|\mathbf{f}^{*}\right\|_{\mathscr{H}}^{2}=\lim _{n \rightarrow \infty} \sum_{j=1}^{n} \sum_{j^{\prime}=1}^{n} f\left(p_{j}\right) \widetilde{a_{j j^{\prime}}} \overline{f\left(p_{j^{\prime}}\right)} .
$$

Here, $\widetilde{a_{j j^{\prime}}}$ is the element of the complex conjugate inverse of the positive definite Hermitian matrix formed by 


$$
a_{j j^{\prime}}=K\left(p_{j}, p_{j^{\prime}}\right) .
$$

In this Proposition, for the uniqueness set of the space, if the reproducing kernel is analytical, then, the criteria will be very simple by the identity theorem of analytic functions. For the Sobolev space cases, we have to consider some dense subset of $E$ for the uniqueness set. Meanwhile, the linearly independence will be easily derived from the integral representations of the kernels.

From the great mathematicians books $[5,6,8,9$, 10], we can find many and many concrete problems among partial differential equations, eigenfunctions, integral transforms and reproducing kernels which are admitting the application of these results.

Acknowledgements This work was supported by Portuguese funds through the CIDMA - Center for Research and Development in Mathematics and Applications, and the Portuguese Foundation for Science and Technology ("FCT-Fundação para a Ciência e a Tecnologia"), within project PEstOE/MAT/UI4106/2014. The third author is supported in part by the Grant-in-Aid for the Scientific Research (C)(2)(No. 24540113).

\section{References}

1. N. Aronszajn, Theory of reproducing kernels, Trans. Amer. Math. Soc., 68 (1950), 337-404.

2. L.P. Castro, H. Fujiwara, M.M. Rodrigues, S. Saitoh, V.K. Tuan, Aveiro discretization method in Mathematics: A new discretization principle, Mathematics without Boundaries: Surveys in Pure Mathematics, Panos Pardalos and Themistocles M. Rassias (edts.), 52 pp. SpringerVerlag, New York (to appear).

3. L.P. Castro, H. Fujiwara, T. Qian, S. Saitoh, How to catch smoothing properties and analyticity of functions by computers?. Mathematics without Boundaries: Surveys in Pure Mathematics, Panos Pardalos and Themistocles M. Rassias (edts.), 15 pp. Springer-Verlag, New York (to appear).

4. L.P. Castro, M.M. Rodrigues, S. Saitoh, A fundamental theorem on linear operator equations using the theory of reproducing kernels, submitted.

5. A. Erdélyi, W. Magnus, F. Oberhettinger, F.G. Tricomi, Tables of Integral Transforms-Vol I, II. Bateman Manuscript Project. California Institute of Technology, McGraw Hill, New York, 1954.

6. I.S. Gradshlein, I.M. Ryzhik, Table of Integrals, Series, and Products, 7th Edition, Elsevier Inc., New York, 2007.

7. H. Hochstadt, Integral Equations, John Wiley and Sons, New York, 1973.

8. A.P. Prudnikov, Yu.A. Brychkov, O.I. Marichev, Integrals and Series, Volume 3: More special functions, Gordon and Breach Publisher, New York, 1990.

9. A.D. Polyanin, Handbook of Linear Partial Differential Equations for Engineers and Scientists, Chapman \& Hall/CRC, Boca Raton, FL, 2002.

10. A.D. Polyanin, V.F. Zaitsev, Handbook of Exact Solutions for Ordinary Differential Equations, CRC Press, Boca Raton, FL, 2003.

11. A.D. Polyanin, A.V. Manzhirov, Handbook of Integral Equations, CRC Press, Boca Raton, FL, 2008.

12. S. Saitoh, Hilbert spaces induced by Hilbert space valued functions, Proc. Amer. Math. Soc., 89 (1983), 74-78.

13. S. Saitoh, Integral Transforms, Reproducing Kernels and their Applications, Pitman Research Notes in Mathematics Series 369, Addison Wesley Longman, Harlow, 1997. 
14. S. Saitoh, Theory of reproducing kernels: Applications to approximate solutions of bounded linear operator functions on Hilbert spaces, Amer. Math. Soc. Transl. Ser, 230, Amer. Math. Soc., Providence, RI, 2010. 